\title{
Posttraumatic parkinsonism would increase the mortality risk in elderly patients with traumatic brain injury
}

\author{
Dorji Harnod $^{1,2}$, Yu-Shu Yen ${ }^{3,4}$, Cheng-Li Lin ${ }^{5,6}$, Tomor Harnod ${ }^{7,8}$, Chia-Hung Kao ${ }^{9,10,11}$ \\ ${ }^{1}$ Department of Emergency and Critical Care Medicine, Fu Jen Catholic University Hospital, ${ }^{2}$ School of Medicine, College of Medicine, Fu Jen \\ Catholic University, New Taipei City; ${ }^{3}$ Department of Neurosurgery, Neurological Institute, Taipei Veterans General Hospital, Taipei; ${ }^{4}$ School of \\ Medicine, National Yang-Ming University, Taipei; ${ }^{5}$ Management Office for Health Data, China Medical University Hospital, Taichung; ${ }^{6}$ College of \\ Medicine, China Medical University, Taichung; ${ }^{7}$ Department of Neurosurgery, Hualien Tzu Chi Hospital, Buddhist Tzu Chi Medical Foundation, \\ Hualien; ${ }^{8}$ College of Medicine, Tzu Chi University, Hualien; ${ }^{9}$ Graduate Institute of Biomedical Sciences, College of Medicine, China Medical \\ University, Taichung; ${ }^{10}$ Department of Nuclear Medicine and PET Center, and Center of Augmented Intelligence in Healthcare, China Medical \\ University Hospital, Taichung; ${ }^{11}$ Department of Bioinformatics and Medical Engineering, Asia University, Taichung \\ Contributions: (I) Conception and design: D Harnod, T Harnod, CH Kao; (II) Administrative support: None; (III) Provision of study materials or \\ patients: CH Kao; (IV) Collection and assembly of data: All authors; (V) Data analysis and interpretation: All authors; (VI) Manuscript writing: All \\ authors; (VII) Final approval of manuscript: All authors. \\ Correspondence to: Chia-Hung Kao, MD. Graduate Institute of Clinical Medicine Science and School of Medicine, College of Medicine, China \\ Medical University, No. 2, Yuh-Der Road, Taichung 404. Email: d10040@mail.cmuh.org.tw; dr.kaochiahung@gmail.com; Tomor Harnod, MD. \\ Department of Neurosurgery, Hualien Tzu Chi Hospital, Buddhist Tzu Chi Medical Foundation, 707, Sec. 3, Chung-Yang Road, Hualien 970. \\ Email: nsha@tzuchi.com.tw.
}

Background: We used data from the National Health Insurance Research Database (NHIRD) of Taiwan to determine whether patients who develop posttraumatic parkinsonism (PTP) after traumatic brain injury (TBI) have an increased mortality risk.

Methods: We analyzed data from the NHIRD of patients aged $\geq 20$ years who had received a diagnosis of and admitted for head injury with TBI (ICD-9-CM 850-854, 959.01) from 2000 to 2012. The TBI cohort was further divided into PTP and posttraumatic non-parkinsonism (PTN) cohorts and compared against a sex-, age-, comorbidity-, and index-date-matched comparison cohort. We calculated the adjusted hazard ratios (aHRs) and $95 \%$ confidence intervals (CIs) of all-cause mortality risk in these cohorts after adjustment for age, sex, and comorbidities.

Results: There were 23,504 and 744 patients enrolled in the PTN cohort and the PTP cohort. Subsequent parkinsonism happened with an incidence of $3.07 \%$ in patients with TBI, and $11.59 \%$ in those aged over 65 years. The PTP cohort ( $\mathrm{aHR}=1.67,95 \%$ CI: 1.47-1.90) other than PTN (aHR =1.37, 95\% CI: 1.29-1.45) cohort had a higher risk of mortality. In ones aged $65-74$ years ( $\mathrm{aHR}=2.08,95 \% \mathrm{CI}$ : 1.41-3.07), there was a more increased mortality risk in patients with PTP when compared to the PTN cohort.

Conclusions: PTP would increase the long-term mortality risk of patients with TBI, especially in whom aged 65-74 years. Our findings provide vital information for clinicians and the government to improve the long-term prognosis of TBI.

Keywords: Cohort study; mortality; National Health Insurance; parkinsonism; traumatic brain injury (TBI)

Submitted Aug 13, 2019. Accepted for publication Nov 19, 2019.

doi: $10.21037 /$ atm.2019.12.04

View this article at: http://dx.doi.org/10.21037/atm.2019.12.04 


\section{Introduction}

Parkinsonism is one of the most common neurodegenerative disorders. Idiopathic parkinsonism, also called Parkinson's disease (PD), with a known prevalence of 60 to 261 patients per 100,000 individuals $(1,2)$, and likely affects $1-2 \%$ of the population aged over 65 years. However, although PD makes up the largest subgroup of all patients with parkinsonism, it is estimated to occupy only approximately half of all parkinsonisms $(3,4)$. Parkinsonism may develop due to many preceding brain insults or diseases. Several hypotheses have been proposed for the pathogenesis of parkinsonism, including genetic predilection, aging, environmental factors, oxidative stress, excitotoxicity, autoimmunity, and trauma (2-4). de Lau et al. studied differences among different types of parkinsonisms and reported that the overall incidence of all types of parkinsonism increases with age and does not differ between men and women, whereas PD particularly seems to have a higher incidence in men than in women (3). Several studies have examined risk factors for PD and mortality risk in patients with PD (5-7); however, there are insufficient data regarding mortality risk and correlated risk factors in patients with parkinsonism after traumatic brain injury (TBI).

TBI is highly prevalent worldwide and is associated with substantial early and long-term mortality. Hsu et al. reported 4,935 in-hospital deaths among 48,792 patients admitted for moderate or severe head injury, with a mortality rate of 10.7 deaths per 100,000 person-years, as per the 2007-2008 total admissions claims dataset of Taiwan (8). Moreover, patients surviving the early stage of TBI usually have a high risk of subsequent disabilities, comorbidities, and neurological deficits $(9,10)$, consequently increasing the medical burden and mortality risk of the patients. We have speculated that TBI would reduce the life expectancy of patients by 6 years after discharge from acute hospitalization (11). However, the actual incidence, extent of increase in mortality risk, and correlated risk factors for mortality of posttraumatic parkinsonism (PTP) in patients with TBI still remain unclear. That how many differences in mortality risk between patients with and without PTP should be investigated to elucidate future treatment strategies for TBI and PTP. In addition, because Taiwanese ethnic and cultural backgrounds are similar to those of the majority of East Asian societies (12), the findings of this study may aid the future development and implementation of other medical care systems in Asian countries.

\section{Methods}

\section{Data source}

The Taiwan National Health Insurance Database (NHIRD) was established and released by Taiwan National Health Research Institutes (Taiwan NHRI). The database contains information regarding health and medical treatment related to ambulatory and inpatient care for nearly $99 \%$ of 23.74 million people in Taiwan (13). In this study, we used the Longitudinal Health Insurance Database 2000 (LHID 2000), which contains the information of 1 million participants randomly selected from the NHIRD, and ensured that the distribution of age and sex matched that of the entire Taiwanese population. The identity of each patient was safeguarded by encrypting the identification number before the data were released. In addition, all diagnoses in the database are coded according to the International Classification of Diseases, Ninth Revision, Clinical Modification (ICD-9-CM) criteria.

The Research Ethics Committee of China Medical University and Hospital in Taiwan approved this study (CMUH104-REC2-115-CR4).

\section{Study population}

We included a TBI cohort of patients aged 20 years or more who were admitted for head injury (ICD-9-CM 850-854 and 959.01) from January 2000 to December 2012. Taiwan NHI guidelines would not accept admission for mild head injury if the patient experiences a head injury with clear consciousness and does not have any intracranial hemorrhage or brain contusion based on brain images. Therefore, the patients' head injuries of this study were qualified as moderate or severe head injuries with TBI. The TBI cohort was divided into PTP cohort and posttraumatic non-parkinsonism (PTN) cohort according to whether patients had received a subsequent diagnosis of parkinsonism (ICD-9-CM codes 332, 332.0, 332.1) after the TBI admission. Since the posttraumatic mortality was largely depended on TBI severity itself, all patients were enrolled after survived from the TBI admission. The date of diagnosis of PTP was defined as the index date. Patients aged below 20 years and those having parkinsonism diagnosis before the TBI admission were excluded. The index date in the comparison cohort was randomly assigned the date as PTP cases. Patients without head injury were selected as the comparison cohort using the same exclusion 


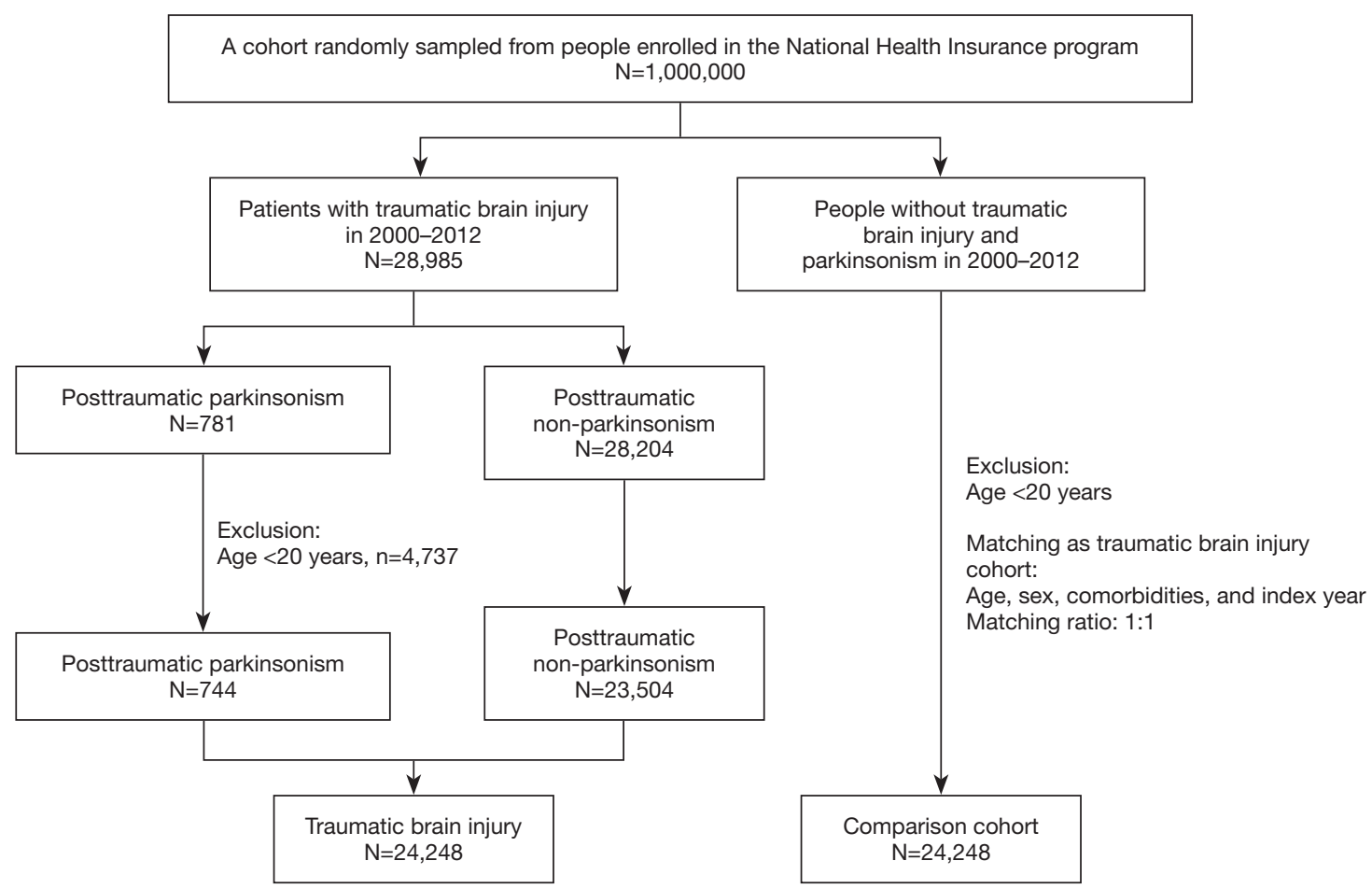

Figure 1 The flow chart shows the selection procedures of participants in this study.

criteria as those used for the TBI cohort and matched 1:1 by sex, age, and index year (Figure 1). Diseases associated with mortality and those diagnosed before the index date were defined as comorbidities, including alcohol-related illness, anxiety disorders, mental disorders, insomnia, depression, stroke, chronic obstructive pulmonary disease (COPD), coronary artery disease (CAD), diabetes, hypertension, hyperlipidemia, asthma, and cancer (including brain tumor). The sociodemographic variables used in this study comprised urbanization level, monthly income [New Taiwan Dollar (NTD); 1.0 USD is approximately 30 NTD], and occupation (white-collar jobs, blue-collar jobs, and others). Person-years were calculated from enrollment till death or the last follow-up (31 December 2013), whichever came first.

\section{Statistical analysis}

In total, 24,248 patients were enrolled in the TBI cohort (23,504 in the PTN cohort and 744 in the PTP cohort). The distribution of age, sex, and comorbidities between TBI and comparison cohorts is expressed as the number and percentage and tested using the chi-square test and $t$-test. The incidence rates of mortality in the cohorts were measured. The cumulative incidence curves of mortality were computed using the Kaplan-Meier method and tested using the log-rank test. The hazard ratio (HR) and 95\% confidence interval (95\% CI) were estimated using the Cox proportional hazards model for evaluating the association between risk factors and mortality. A multivariate Cox proportional hazards model was used to calculate adjusted HRs after adjustment for variables found to be statistically significant in the univariable model. Analysis of stratification by age, sex, and comorbidity was performed to explore the association of head injury and PTP with mortality in a specific population. All statistical analyses were performed using SAS statistical software, version 9.4 (SAS Institute Inc., Cary, North Carolina, USA). Statistical significance was determined using two-tailed tests $(\mathrm{P}<0.05)$.

\section{Data availability statement}

The dataset used in this study belongs to the Taiwan Ministry of Health and Welfare (MOHW). Any researcher interested in accessing this dataset can submit an application form to the MOHW requesting access. Please contact the staff of MOHW (Email: stcarolwu@mohw.gov.tw) for further assistance. Taiwan MOHW address: No. 488, Sec. 6, 
Zhongxiao E. Rd., Nangang Dist., Taipei City 115. Phone: +886-2-8590-6848.

\section{Results}

The mean [standard deviation (SD)] ages of patients were 46.9 (18.0), 46.9 (18.4), 46.2 (18.0) and 70.0 (14.5) in the comparison, total TBI, PTN, and PTP cohorts, respectively. There were $56.3-60.4 \%$ men in the 4 cohorts. Parkinsonism happened with an incidence of $3.1 \%$ (744 out of 24,248 ) in patients with TBI, and likely affected $11.6 \%$ (548 out of 4,728 ) of posttraumatic patients aged over 65 years. Patients in the total TBI cohort tended to reside in higher urbanized areas than comparisons (59.5\% vs. $50.3 \%)$. The monthly incomes in total TBI and comparison cohorts were $43.6 \%$ and $44.3 \%$ with $<15,000$ NTD, 25.8\% and $29.3 \%$ with 15,000-19,999 NTD, and $30.6 \%$ and $26.4 \%$ with $\geq 20,000$ NTD. The distributions of white-collar occupation (53.5\% vs. $43.8 \%)$ and blue-collar occupation $(36.5 \%$ vs. $44.9 \%$ ) were noted in total TBI and comparison cohorts. Compared with the comparison cohort, a significantly higher proportion of patients in the TBI cohort had alcohol-related illness, mental disorders, and depression. However, a lower proportion of patients in the TBI cohort had stroke (Table 1).

The cumulative incidence of mortality in the PTP, PTN, and comparison cohorts is illustrated in Figure 2. There were significant differences in the cumulative incidence curves between the 3 populations, and the $\mathrm{P}$ value for $\log$ rank test was less than 0.001 (Figure 2). The patients in total TBI cohort, the patients with PTN, the patients with PTP, and comparisons had mortality rates of $19.7,18.2,80.0$, and 14.0 per 1,000 person-years, respectively. Compared with the comparison cohort, the adjusted HRs (95\% CI) for the mortality of patients with TBI, PTN, and PTP were 1.39 (1.32-1.48), 1.37 (1.29-1.45), and 1.67 (1.471.90), respectively. The higher monthly income $(\geq 20,000$ NTD) and middle monthly income (15,000-19,999 NTD) had an adjusted HR of 1.67 (95\% CI: 1.55-1.80) and an adjusted HR of 1.59 (95\% CI: 1.46-1.73) for mortality compared with those lower monthly income $(<15,000$ NTD). The adjusted HR was 1.15 -fold for those living at lowest urbanized areas, compared with those living at highest urbanized areas (95\% CI: 1.06-1.25), and 1.27fold for parents with other occupations compared with those parents with white collar occupations (95\% CI: 1.16-1.39). Advanced age over 65 years, male sex, alcoholrelated illness, mental disorders, stroke, COPD, CAD, diabetes mellitus, hypertension, asthma, and cancer were significantly positively associated with mortality. However, anxiety disorders, insomnia, and hyperlipidemia were significantly negatively associated with mortality (Table 2 ).

The associations of total TBI, PTN, and PTP cohorts with mortality were stratified by age, sex, and comorbidity, as demonstrated in Table 3. By the analyzed results in Table 2, we divided the elder age group (over 65 years) into $65-74$ years and $\geq 75$ years for further analysis. The incidences of developing parkinsonism after TBI were $1.17 \%$ (9 in 771 ), $5.66 \%$ (30 in 530 ), and $15.37 \%$ (244 in 1,588 ) in the patients aged $40-64$ years, $65-74$ years, and $\geq 75$ years. Compared with the comparison cohort, PTN was significantly associated with higher mortality in each subgroup of age (adjusted HRs: 1.87, 1.32, and 1.26, for 40-64 years, 65-74 years, and $\geq 75$ years, respectively). Compared with the comparison cohort, PTP cohort had increased mortality in each subgroup of age (adjusted HRs: $1.61,2.65$, and 1.56 , for $40-64$ years, $65-74$ years, and $\geq 75$ years, respectively) with a $\mathrm{P}$ for interaction $<0.001$. PTP was associated with a specifically higher mortality in patients aged 65-74 years. Compared with the comparison cohort, the 3 posttraumatic cohorts showed significant positive associations with increased mortality risk in both men and women, and sex with a P for interaction $<0.001$. Compared with the comparison cohort, TBI and PTN groups had adjusted HRs (95\% CI) of mortality of 1.50 (1.30-1.74) and $1.44(1.24-1.67)$, respectively, in patients without comorbidity. Compared with the comparison cohort, TBI, PTN, and PTP cohorts had adjusted HRs (95\% CI) of mortality of 1.40 (1.31-1.48), 1.34 (1.26-1.43), and 1.78 (1.57-2.02), respectively, in patients with any of the comorbidities (Table 3).

The differences of mortality risk between PTP and PTN cohorts were further compared with stratification of age, sex, and comorbidity, as shown in Table 4. The PTP cohort had a 1.27 -fold mortality risk than that of the PTN cohort. Specifically, in patients aged $65-74$ years and those older than 75 years, those with PTP had 2.08- and 1.25 -fold mortality risks, respectively, than did those of patients with PTN. Compared with the PTN cohort, women and men with PTP had adjusted HRs (95\% CI) of mortality of $1.29(1.05-1.59)$ and $1.23(1.04-1.45)$, respectively. Among patients without any comorbidity, there was no significant association between PTP and mortality. However, patients with PTP had a significantly higher mortality than did patients with any comorbidity, with an adjusted HR (95\% CI) of 1.40 (1.23-1.59) (Table 4). 
Table 1 Distribution of age, sex, and comorbidities between patients with traumatic brain injury with or without parkinsonism, and comparison cohorts

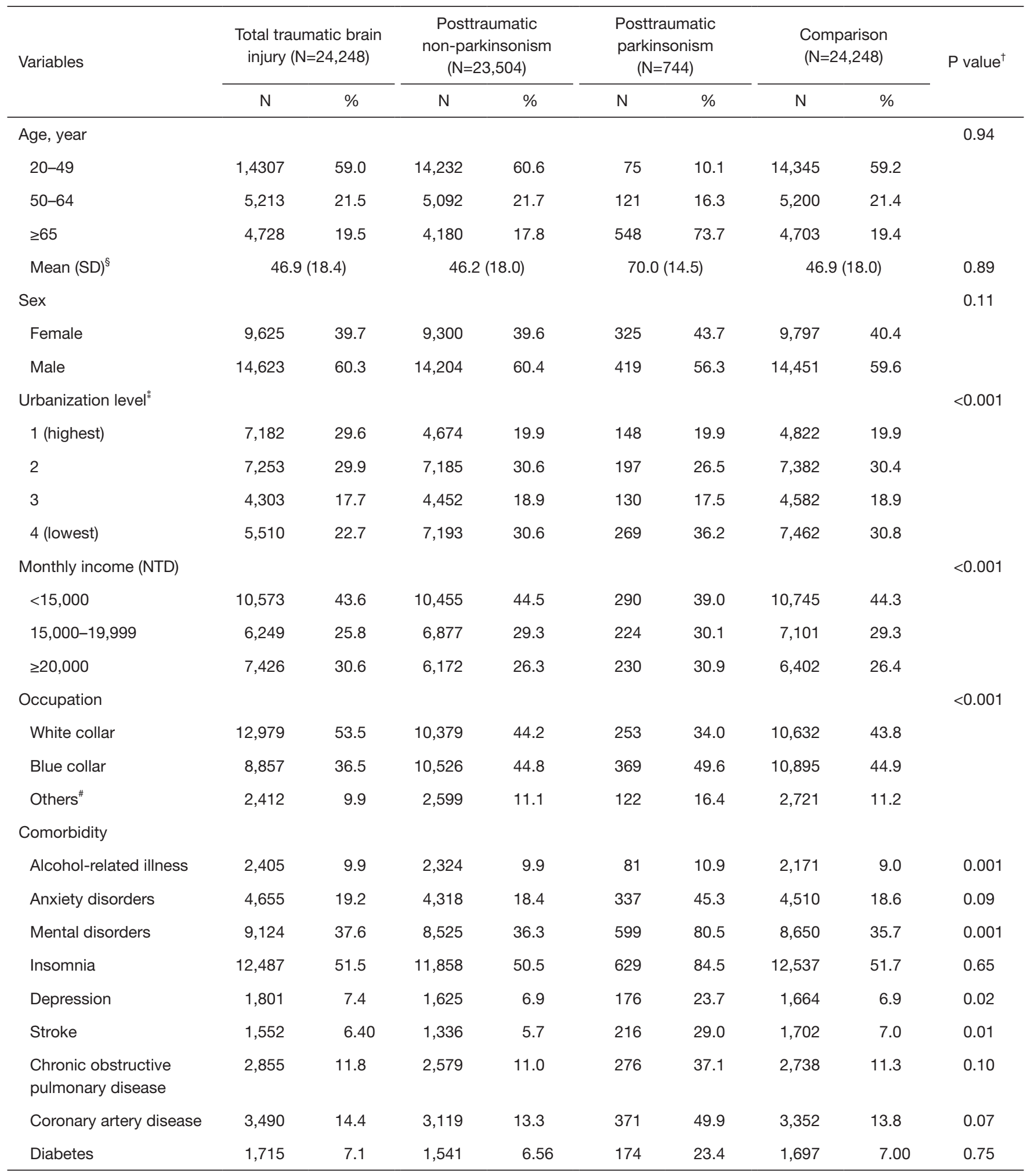

Table 1 (continued) 
Table 1 (continued)

\begin{tabular}{|c|c|c|c|c|c|c|c|c|c|}
\hline Variables & \multicolumn{2}{|c|}{$\begin{array}{l}\text { Total traumatic brain } \\
\text { injury }(\mathrm{N}=24,248)\end{array}$} & \multicolumn{2}{|c|}{$\begin{array}{c}\text { Posttraumatic } \\
\text { non-parkinsonism } \\
(\mathrm{N}=23,504)\end{array}$} & \multicolumn{2}{|c|}{$\begin{array}{l}\text { Posttraumatic } \\
\text { parkinsonism } \\
(\mathrm{N}=744)\end{array}$} & \multicolumn{2}{|c|}{$\begin{array}{l}\text { Comparison } \\
(\mathrm{N}=24,248)\end{array}$} & $P$ value ${ }^{\dagger}$ \\
\hline Hypertension & 6,775 & 27.9 & 6,218 & 26.5 & 557 & 74.9 & 6,741 & 27.8 & 0.73 \\
\hline Hyperlipidemia & 4,287 & 17.7 & 3,998 & 17.0 & 289 & 38.8 & 4,181 & 17.2 & 0.20 \\
\hline $\begin{array}{l}\text { Cancer (including brain } \\
\text { tumor) }\end{array}$ & 511 & 2.11 & 483 & 2.1 & 28 & 3.8 & 553 & 2.3 & 0.19 \\
\hline
\end{tabular}

Chi-square test. ${ }^{\S}, t$-test; ${ }^{\dagger}$, total traumatic brain injury cohort vs. comparison cohort; ${ }^{*}$, other occupations included primarily retired, unemployed, or low income populations; ; , the urbanization level was categorized by the population density of the residential area into 4 levels, with level 1 as the most urbanized and level 4 as the least urbanized. NTD: New Taiwan Dollars per month. One New Taiwan Dollar equals 0.03 US Dollar. SD, standard deviation.

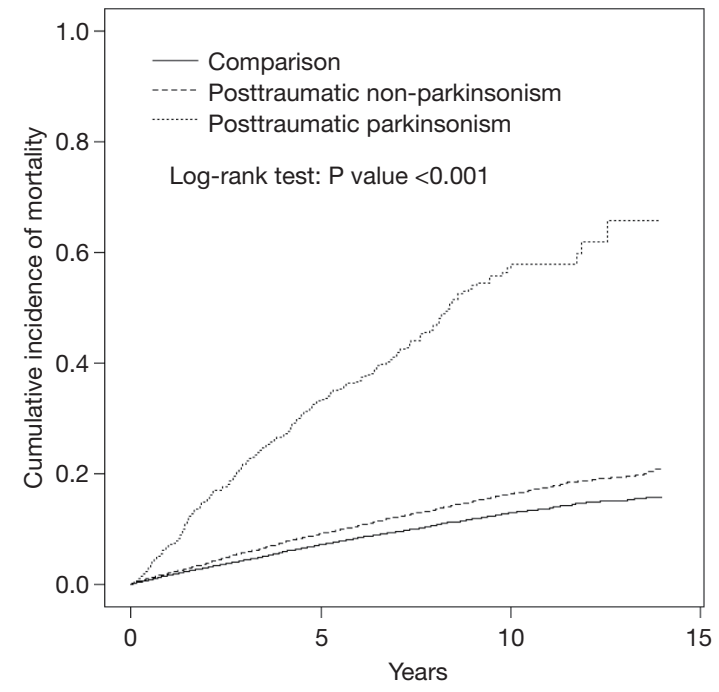

Figure 2 Comparison of cumulative incidence of mortality among patients with posttraumatic parkinsonism, those with traumatic brain injury without parkinsonism, and comparisons.

\section{Discussion}

In contrast to the popular belief that approximately $1-2 \%$ of elderly individuals develop idiopathic parkinsonism (2), our results revealed that $3.1 \%$ in patients with TBI and $11.6 \%$ in those aged over 65 years would develop subsequent parkinsonism after TBI. Moreover, Taiwanese patients with PTP, those with TBI without PTP, and comparisons had respective mortality rates of $80.0,18.2$, and 14.0 per 1,000 person-years. Therefore, patients with TBI and subsequent parkinsonism had a generally 1.67 -fold mortality risk than did the comparison cohort, whereas those with TBI without PTP had a less (1.37-fold) mortality risk than did the comparison cohort. Furthermore, the patients with PTP and aged 65-74 years would have specifically significant increased mortality risks other than patients with other ages. Rugbjerg et al. conducted a study similar to ours and reported a $50 \%$ increased risk of parkinsonism in a 10 -year duration in patients undergoing hospital treatment for head injuries (14). It is difficult to compare the results of our large-scale study with a 14-year follow-up duration to the results of other studies because these studies enrolled patients with different types of parkinsonism and had a limited follow-up duration $(2,7,10)$. However, this large study population, which excluded patients with mild head injury who were treated through outpatient services, demonstrated that development of parkinsonism in elderly patients after TBI would much often occur and could be considered as a negative predictor for their long-term survival.

Parkinsonism is a progressive degenerative disease, the cumulative incidence of new diagnosis and mortality rate of these patients should gradually increase with an increase in their age or posttraumatic duration. Our results documented a trend of increasing incidence of PTP with patients' age; with $1.17 \%, 5.66 \%$, and $15.37 \%$ in the patients aged 40-64 years, $65-74$ years, and $\geq 75$ years, respectively. However, we found that there was a 2.65 -fold mortality risk in the ones with PTP with age of 65-74 years; other than a 1.56 -fold mortality risk in those aged $\geq 75$ years and a 1.61 fold mortality risk in those aged $40-64$ years. The highest mortality risk in the 65-74-year-old subgroup of PTP 
Table 2 Incidence and hazard ratio for mortality with different risk factors

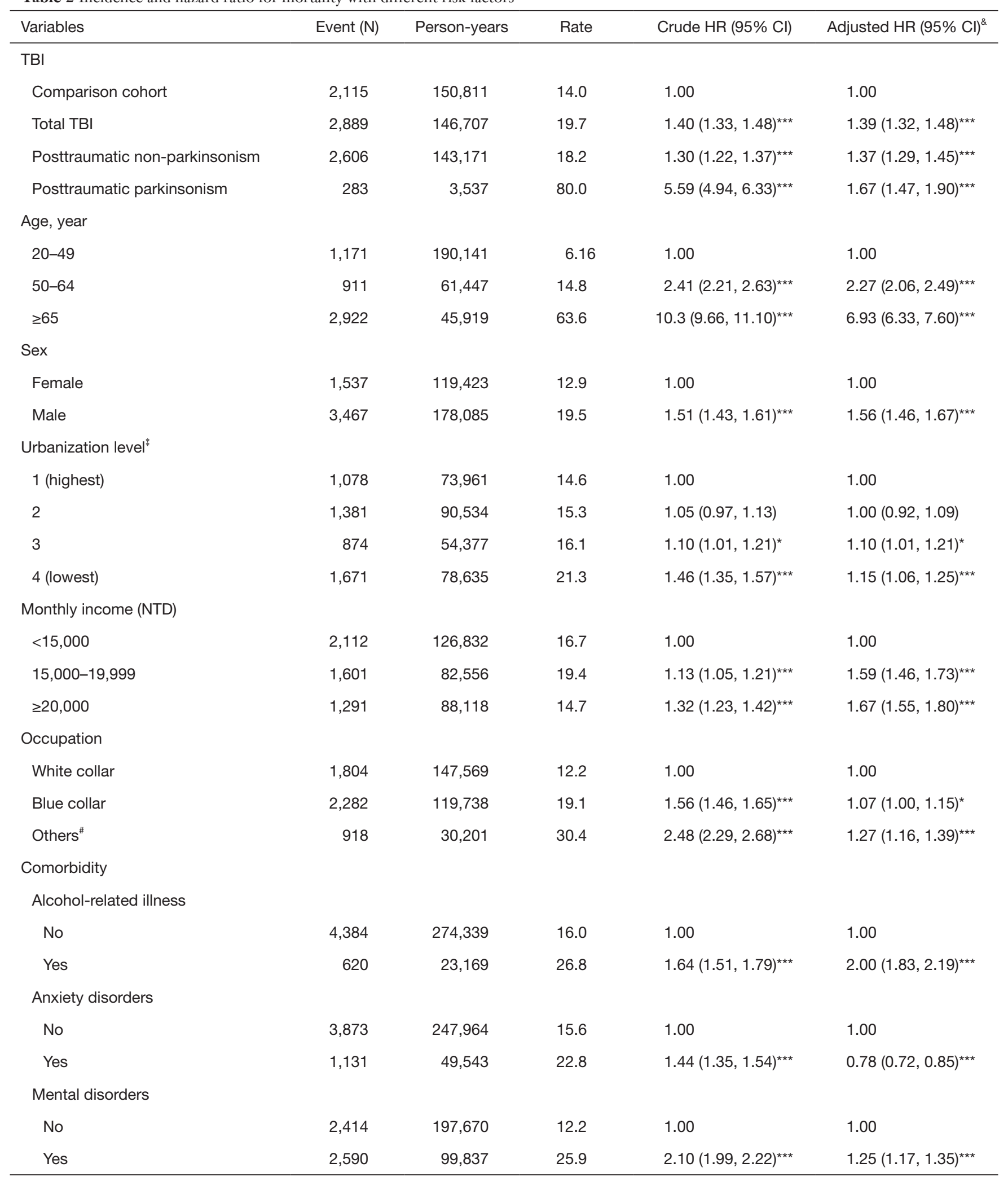

Table 2 (continued) 
Table 2 (continued)

\begin{tabular}{|c|c|c|c|c|c|}
\hline Variables & Event $(\mathrm{N})$ & Person-years & Rate & Crude HR (95\% Cl) & Adjusted HR $(95 \% \mathrm{Cl})^{i^{\circledR}}$ \\
\hline No & 1,941 & 159,608 & 12.2 & 1.00 & 1.00 \\
\hline Yes & 3,063 & 137,899 & 22.2 & $1.80(1.70,1.90)^{\star \star \star}$ & $0.89(0.83,0.95)^{\star \star \star}$ \\
\hline \multicolumn{6}{|c|}{ Depression } \\
\hline Yes & 412 & 18,098 & 22.8 & $1.36(1.23,1.51)^{\star \star \star}$ & $1.05(0.94,1.18)$ \\
\hline \multicolumn{6}{|l|}{ Stroke } \\
\hline No & 3,866 & 282,920 & 13.7 & 1.00 & 1.00 \\
\hline Yes & 1,138 & 14,587 & 78.0 & $5.63(5.27,6.02)^{\star \star \star}$ & $1.80(1.67,1.93)^{\star \star \star}$ \\
\hline Yes & 1,481 & 289,193 & 52.5 & $3.97(3.73,4.22)^{\star \star \star}$ & $1.25(1.16,1.35)^{\star \star \star}$ \\
\hline \multicolumn{6}{|c|}{ Coronary artery disease } \\
\hline No & 3,285 & 261,956 & 12.5 & 1.00 & 1.00 \\
\hline Yes & 1,719 & 35,551 & 48.4 & $3.82(3.60,4.05)^{\star \star \star}$ & $1.12(1.04,1.20)^{\star \star \star}$ \\
\hline \multicolumn{6}{|c|}{ Diabetes } \\
\hline No & 4012 & 280,074 & 14.3 & 1.00 & 1.00 \\
\hline Yes & 992 & 17,433 & 56.9 & $3.92(3.66,4.20)^{\star \star \star}$ & $1.73(1.61,1.87)^{\star \star \star}$ \\
\hline Yes & 1,168 & 44,888 & 26.0 & $1.69(1.58,1.81)^{\star \star \star}$ & $0.66(0.62,0.71)^{\star \star \star}$ \\
\hline \multicolumn{6}{|l|}{ Asthma } \\
\hline No & 4,277 & 278,836 & 15.3 & 1.00 & 1.00 \\
\hline Yes & 727 & 18,671 & 38.9 & $2.50(2.31,2.70)^{\star \star \star}$ & $1.15(1.05,1.25)^{\star \star}$ \\
\hline \multicolumn{6}{|c|}{ Cancer (including brain tumor) } \\
\hline No & 4,688 & 293,249 & 16.0 & 1.00 & 1.00 \\
\hline Yes & 316 & 4,258 & 74.2 & $4.51(4.03,5.06)^{\star \star \star}$ & $2.56(2.28,2.88)^{\star \star \star}$ \\
\hline
\end{tabular}

\&, variables found to be statistically significant in the univariate model were further included in the multivariate model; ", other occupations included primarily retired, unemployed, or low income populations; *, the urbanization level was categorized by the population density of the residential area into 4 levels, with level 1 as the most urbanized and level 4 as the least urbanized; ${ }^{\star}, \mathrm{P}<0.05 ;{ }^{\star \star}, \mathrm{P}<0.01 ;{ }^{\star \star \star}, \mathrm{P}<0.001$. NTD: New Taiwan Dollars per month. One New Taiwan Dollar equals 0.03 US Dollar. TBI, traumatic brain injury; Rate, per 1,000 personyears; HR, relative hazard ratio. 
Table 3 Comparison of incidence and hazard ratio for mortality stratified by age, sex, and comorbidity between patients with traumatic brain injury with or without parkinsonism, and comparison cohorts

\begin{tabular}{|c|c|c|c|c|c|c|c|c|c|c|c|}
\hline \multirow[b]{2}{*}{ Variables } & \multirow{2}{*}{\multicolumn{2}{|c|}{$\begin{array}{c}\text { Comparison } \\
\text { cohort } \\
(\mathrm{N}=24,248)\end{array}$}} & \multicolumn{9}{|c|}{ Posttraumatic brain injury (TBI) } \\
\hline & & & \multicolumn{3}{|c|}{ Total TBI $(\mathrm{N}=24,248)$} & \multicolumn{3}{|c|}{$\begin{array}{l}\text { Posttraumatic non-parkinsonism } \\
\qquad(\mathrm{N}=23,504)\end{array}$} & \multicolumn{3}{|c|}{$\begin{array}{l}\text { Posttraumatic parkinsonism } \\
\qquad(\mathrm{N}=744)\end{array}$} \\
\hline \multicolumn{12}{|l|}{ Age, year } \\
\hline $65-74$ & 381 & 12.4 & 530 & 17.3 & $1.36(1.19,1.55)^{\star \star \star}$ & 500 & 16.6 & $1.32(1.15,1.51)^{\star \star \star}$ & 30 & 51.5 & $2.65(1.80,3.90)^{\star \star \star}$ \\
\hline$\geq 75$ & 1,334 & 56.7 & 1,588 & 70.9 & $1.30(1.21,1.40)^{\star \star *}$ & 1,344 & 67.3 & $1.26(1.17,1.37)^{\star \star \star}$ & 244 & 100.5 & $1.56(1.36,1.80)^{\star \star \star}$ \\
\hline $\mathrm{P}$ for interaction & - & - & - & - & $<0.001$ & - & - & $<0.001$ & - & - & - \\
\hline \multicolumn{12}{|l|}{ Sex } \\
\hline $\mathrm{P}$ for interaction & - & - & - & - & $<0.001$ & - & - & 0.02 & - & - & - \\
\hline \multicolumn{12}{|l|}{ Comorbidity } \\
\hline None & 303 & 5.62 & 431 & 8.33 & $1.50(1.30,1.74)^{\star \star \star}$ & 429 & 8.30 & $1.44(1.24,1.67)^{\star \star \star}$ & 2 & 35.6 & $1.42(0.35,5.73)$ \\
\hline With any one & 1,812 & 18.7 & 2,458 & 25.9 & $1.40(1.31,1.48)^{\star * \star}$ & 2,177 & 23.8 & $1.34(1.26,1.43)^{\star \star \star}$ & 281 & 80.7 & $1.78(1.57,2.02)^{\star \star \star}$ \\
\hline $\mathrm{P}$ for interaction & - & - & - & - & 0.41 & - & - & 0.63 & - & - & - \\
\hline
\end{tabular}

\footnotetext{
${ }^{\&}$, variables found to be statistically significant in the univariate model were further included in the multivariate model; ${ }^{\star \star}, \mathrm{P}<0.01 ;{ }^{\star \star \star}$,
}

$\mathrm{P}<0.001$. TBI, traumatic brain injury; Rate, per 1,000 person-years; HR, relative hazard ratio.

Table 4 Comparison of hazard ratio for mortality stratified by age, sex, and comorbidities between patients with and without posttraumatic parkinsonism

\begin{tabular}{|c|c|c|}
\hline Variables & \multicolumn{2}{|c|}{ Adjusted $\mathrm{HR}^{\&}(95 \% \mathrm{Cl})$} \\
\hline All & 1.00 & $1.27(1.11,1.44)^{\star \star \star}$ \\
\hline \multicolumn{3}{|l|}{ Age, year } \\
\hline $40-64$ & 1.00 & $0.85(0.43,1.67)$ \\
\hline$\geq 75$ & 1.00 & $1.25(1.08,1.43)^{\star \star \star}$ \\
\hline \multicolumn{3}{|l|}{ Sex } \\
\hline Female & 1.00 & $1.29(1.05,1.59)^{\star \star}$ \\
\hline Male & 1.00 & $1.23(1.04,1.45)^{\star \star}$ \\
\hline With any one & 1.00 & $1.40(1.23,1.59)^{\star \star \star}$ \\
\hline
\end{tabular}

\footnotetext{
\&, variables found to be statistically significant in the univariate model were further included in the multivariate model; ${ }^{* *}, \mathrm{P}<0.01 ;{ }^{\star \star *}$,
} $\mathrm{P}<0.001$. HR, relative hazard ratio; $\mathrm{Cl}$, confidence interval. 
cohort is out of our though before. It might be due to that development of subsequent parkinsonism and patients' allcause mortality are not only caused from the consequence of TBI, but also from stoke, other neuro-degenerative or systemic diseases in elder patients. Although we already controlled various comorbidities for adjustment of analysis, including alcohol-related illness, anxiety disorders, mental disorders, insomnia, depression, stroke, COPD, CAD, diabetes, hypertension, hyperlipidemia, asthma, and cancer (including brain tumor) in this study, some other predisposing factors for developing parkinsonism and all-cause mortality could be existed in our patients. The mortality of patients with subsequent parkinsonism after TBI is associated with a lot of complex health conditions, those would together result in the increase of mortality risk, not the PTP only. However, what we found the highest mortality risk in the 65-74-year-old subgroup has implied that age should not being the most powerful factor for the mortality in patients with PTP.

Parkinsonism is a disorder resulting from the deficiency of dopamine. The pathogenesis of and predisposing factors for secondary parkinsonism still remain unclear because the possible mechanisms underlying the development of parkinsonism are more multifactorial and complicated than those underlying the development of idiopathic parkinsonism. These include external factors from different brain insults and internal factors that show interactions in a genetically predisposed individual $(15,16)$. First, TBI could immediately damage the thalamus and other deep brain nuclei or it may induce chronic inflammatory encephalopathy $(2,17)$. Second, TBI could induce an inflammatory cascade and lead to the accumulation of $\alpha$-synuclein and tau, which are the major components of Lewy bodies responsible for developing parkinsonism (18-20). In addition, through disruption of the blood-brain barrier or through mitochondrial dysfunction, TBI may contribute to the development of PTP $(21,22)$. All of these could result posttraumatic patients developing PTP owing to the interaction of multiple mechanisms and lead to a higher mortality risk.

Our data also suggest that multiple comorbid diseases may act together to increase the risk of parkinsonism and the related mortality risk. In addition to cancer and brain tumor, which can directly increase the mortality risk of patients, comorbidities, such as alcohol-related illness, mental disorders, stroke, COPD, CAD, diabetes, hypertension, and asthma, could directly or indirectly increase the mortality risk in patients with PTP. Among these factors, stroke, CAD, diabetes, and hypertension are vascular-related risk factors, and vascular parkinsonism could be attributed to disrupted connections among the basal ganglia, thalamus, motor cortex, and brain stem $(23,24)$. Alcohol-related illness, COPD, and asthma can be predisposing factors for increasing chronic inflammation. Those comorbidities could further interact with naturally clinical neuro-pathological progress, resulting in the development of parkinsonism and increasing the risk of mortality in patients.

In this study, we enrolled patients with first-ever or recurrent admission for TBI to analyze the possible correlation of PTP and subsequent long-term all-cause mortality. However, this study has several limitations and unadjusted bias. First, the identities of patients were anonymized to avoid possible direct contact or access to their detailed medical records in the NHIRD. Therefore, we could not analyze the type and severity of TBIs at admission, the burden or severity of parkinsonism, or medication for their TBI and PTP. The posttraumatic mortality risk was for sure largely depended on TBI severity, and its' confounding effect was minimized as possible in this study by enrolling the participants after they survived from the TBI admission. Moreover, although the severity of idiopathic parkinsonism-PD is convincible measured with the Unified Parkinson's Disease Rating Scale (UPDRS) for years, the strength of UPDRS applying over other types of parkinsonisms have not well accepted for utilization $(25,26)$. The posttraumatic handicaps by TBI might mislead the severity measurement of parkinsonism for further categorization and analysis. In addition, we could not rule out any long-term mortality that was caused by the severe handicapped condition after TBI, or indirectly, by other diseases. Second, several socioeconomic factors, including urbanization level, monthly income, and occupation were categorized and analyzed for the mortality risk in this study. We did not find obvious differences of mortality risk between the subgroups of these factors. However, these factors could not totally correlate with whether a posttraumatic patient would get good care from their family or government to secure the patient's long-term survival. Finally, there is a rare possibility of miscoding in the NHIRD, although the NHI program involves thorough quarterly reviews and false claims are heavily penalized to ensure that the files are accurate. Moreover, although our study design included and adjusted numerous confounding factors, unmeasured or unknown confounders may have generated a bias. However, this study was statistically 
sufficient to demonstrate the incidence, mortality risk, and specific risk factors for mortality in patients with PTP. These results provide us the importance and future strategy of improvement of posttraumatic care in the aged population.

\section{Conclusions}

The long-term all-cause mortality risk of patients with TBI who develop PTP is significantly higher than the corresponding risk of patients without PTP, especially in those with aged 65-74 years. Our findings might provide vital information for clinicians and the government to improve long-term care strategies in elderly patients with TBI. We are looking forward to more large-scale studies around the world to clarify the global impacts of PTP on elderly patients with TBI.

\section{Acknowledgments}

Funding: This study is supported in part by Taiwan Ministry of Health and Welfare Clinical Trial Center (MOHW108TDU-B-212-133004), China Medical University Hospital, Academia Sinica Stroke Biosignature Project (BM10701010021), MOST Clinical Trial Consortium for Stroke (MOST 108-2321-B-039-003-), Tseng-Lien Lin Foundation, Taichung, and Katsuzo and Kiyo Aoshima Memorial Funds, Japan.

\section{Footnote}

Conflicts of Interest: The authors have no conflicts of interest to declare.

Ethical Statement: The authors are accountable for all aspects of the work in ensuring that questions related to the accuracy or integrity of any part of the work are appropriately investigated and resolved. The Research Ethics Committee of China Medical University and Hospital in Taiwan approved this study (CMUH104REC2-115-CR4).

\section{References}

1. Benito-León J, Bermejo-Pareja F, Rodríguez J, et al. Prevalence of PD and other types of parkinsonism in three elderly populations of central Spain. Mov disord 2003;18:267-74.
2. Lee PC, Bordelon Y, Bronstein J, et al. Traumatic brain injury, paraquat exposure, and their relationship to Parkinson disease. Neurology 2012;79:2061-6.

3. de Lau LM, Giesbergen PC, de Rijk MC, et al. Incidence of parkinsonism and Parkinson disease in a general population: the Rotterdam Study. Neurology 2004;63:1240-4.

4. Mitchell SL, Rockwood K. Defining parkinsonism in the Canadian Study of Health and Aging. Int Psychogeriatr 2001;13 Supp 1:107-13.

5. Eric Nyam TT, Ho CH, Wang YL, et al. The Risk of Traumatic Brain Injury Occurring Among Patients with Parkinson Disease: A 14-Year Population-Based Study. World Neurosurg 2018;113:e328-e335.

6. Weintraub D, Chiang C, Kim HM, et al. Association of Antipsychotic use with mortality risk in patients with PD. JAMA Neurol 2016;73:535-41.

7. Mitchell SL, Rockwood K. The association between parkinsonism, Alzheimer's disease, and mortality: a comprehensive approach. J Am Geriatr Soc 2000;48:422-5.

8. Hsu IL, Li CY, Chu DC, et al. An epidemiological analysis of head injuries in Taiwan. Int J Environ Res Public Health 2018. doi: 10.3390/ijerph15112457.

9. Centers for Disease Control and Prevention. Report to Congress. Traumatic brain injury in the United States: epidemiology and rehabilitation. Atlanta: National Center for Injury Prevention and Control, Division of Unintentional Injury Prevention, 2015.

10. Perry DC, Sturm VE, Peterson MJ, et al. Association of traumatic brain injury with subsequent neurological and psychiatric disease: a meta-analysis. J Neurosurg 2016;124:511-26.

11. Ventura T, Harrison-Felix C, Carlson N, et al. Mortality after discharge from acute care hospitalization with traumatic brain injury: a population-based study. Arch Phys Med Rehabil 2010;91:20-9.

12. Ying YW, Han M. Cultural orientation in Southeast Asian American young adults. Cultur Divers Ethnic Minor Psychol 2008;14:29-37.

13. National Health Insurance Research Database. Available online: http://nhird.nhri.org.tw/en/index.html (accessed on 19 March 2019).

14. Rugbjerg K, Ritz B, Korbo L, et al. Risk of Parkinson's disease after hospital contact for head injury: population based case-control study. BMJ 2008;337:a2494.

15. Delamarre A, Meissner WG. Epidemiology, environmental risk factors and genetics of Parkinson's disease. Presse Med 2017;46:175-81. 
16. Ascherio A, Schwarzschild MA. The epidemiology of Parkinson's disease: risk factors and prevention. Lancet Neurol 2016;15:1257-72.

17. Lee MS, Marsden CD. Movement disorders following lesions of the thalamus or subthalamic region. Mov Disord 1994;9:493-507.

18. Smith DH, Uryu K, Saatman KE, et al. Protein accumulation in traumatic brain injury. Neuromolecular Med 2003;4:59-72.

19. Lenzlinger PM, Morganti-Kossmann MC, Laurer HL, et al. The duality of the inflammatory response to traumatic brain injury. Mol Neurobiol 2001;24:169-81.

20. Schmidt OI, Heyde CE, Ertel W, et al. Closed head injury- an inflammatory disease? Brain Res Brain Res Rev 2005;48:388-99.

21. Frantseva M, Perez Velazquez JL, Tonkikh A, et al.

Cite this article as: Harnod D, Yen YS, Lin CL, Harnod T, Kao CH. Posttraumatic parkinsonism would increase the mortality risk in elderly patients with traumatic brain injury. Ann Transl Med 2019;7(23):734. doi: 10.21037/atm.2019.12.04
Neurotrauma/ neurodegeneration and mitochondrial dysfunction. Prog Brain Res 2002;137:171-6.

22. Unterberg AW, Stover J, Kress B, et al. Edema and brain trauma. Neuroscience 2004;129:1021-9.

23. Winikates J, Jankovic J. Clinical correlates of vascular parkinsonism. Arch Neurol 1999;56:98-102.

24. Zijlmans JC. The role of imaging in the diagnosis of vascular parkinsonism. Neuroimaging Clin N Am 2010;20:69-76.

25. Keezer MR, Wolfson C, Postuma RB. Age, gender, comorbidity, and the MDS-UPDRS: results from a population-based study. Neuroepidemiology 2016;46:222-7.

26. Movement Disorder Society Task Force on Rating Scales for Parkinson's Disease. The Unified Parkinson's Disease Rating Scale (UPDRS): status and recommendations. Mov Disord 2003;18:738-50. 